\title{
UNA MIRADA BIBLIOGRÁFICA SOBRE LA INFLUENCIA DE LA PLIOMETRÍA EN EL TREN INFERIOR EN BALONCESTO.
}

\author{
A BIBLIOGRAPHIC REVIEW ABOUT PLYOMETRICS IN THE LOWER TRAIN IN \\ BASKETBALL.
}

Cómo citar: Martínez, W., López, F., Acosta, P., \& Sanabria, Y. (2019). Una mirada bibliográfica sobre la influencia de la pliometría en el tren inferior en baloncesto. R. Actividad fis. y deporte. 6 (1): 179-193.

Artículo de acceso abierto publicado por: Revista Digital: Actividad Física y Deporte, bajo una licencia Creative Commons CC BY-NC 4.0.

\section{Winnen Julián Martínez Cubides}

Lic. en Educación Física, Recreación y Deportes. Maestrante en Pedagogía de la Cultura Física.

wjmartinez@jdc.edu.co.

\section{Fernando Antonio López López}

Lic. en Educación Física, Recreación y Deporte. UPTC. Maestrante en Pedagogía de la Cultura Física. Loztrainer2005@yahoo.es

\section{Paulo Jonathan Acosta Tova}

Dr. (Ph.D): Educación Deportiva y Ciencias del Deporte. Lic. en Educación Física, Recreación y Deporte. Mg. Pedagogía de la cultura física. Docente Universidad Pedagógica y tecnológica de ColombiaUPTC.

Paulo.acosta@uptc.edu.co

\section{Yofre Danilo Sanabria Arguello.}

Dr. (Ph.D): Educación deportiva y Ciencias del deporte. Mg. Pedagogías de la Cultura Física. Docente Universidad Pedagógica y Tecnológica de Colombia-UPTC.

Yofre.sanabria@uptc.edu.co.

\section{RESUMEN}

Introducción: El baloncesto es uno de los deportes que más se practica a nivel colegiado, universitario, profesional y amateur combinando acciones acíclicas y saltos durante los partidos, con intensidades altas, medias y bajas. Por tal razón, la potencia del tren inferior en los saltos verticales utilizados por los deportistas para ganar rebotes ofensivos, defensivos y realizar lanzamientos a canasta de media distancia y 3 puntos, son una parte fundamental dentro del desarrollo de esta modalidad deportiva; en consecuencia, el método de entrenamiento pliométrico puede ser determinante para el incremento de los niveles de la potencia en la capacidad de salto durante la competencia. Objetivo General: Indagar los métodos de entrenamiento pliométrico sobre la potencia del tren inferior de las investigaciones ya culminadas en baloncesto y analizar el resultado obtenido en función de la misma. Metodología: Es una 
investigación con enfoque cualitativo y diseño descriptivo, porque el interés del autor, es obtener información precisa para analizar y comparar la efectividad de los métodos de entrenamiento pliométrico. Resultados: Se fundamentaron en artículos provenientes de las bases de datos, tesis y libros correlacionados con programas de entrenamiento pliométrico a nivel nacional e internacional en la modalidad de baloncesto masculino, que sustentan efectos positivos en la potencia del tren inferior de acuerdo a las semanas planificadas por las investigaciones encontradas. Conclusión: La revisión bibliográfica en baloncesto masculino pretende ser un punto de partida para tener conocimientos avanzados en cuanto a la pliometría, potencia del tren inferior, salto vertical, los instrumentos utilizados y su eficacia durante las sesiones de entrenamiento en el baloncesto masculino.

Palabras Clave: Potencia del tren inferior, Pliometría, Baloncesto.

\section{ABSTRACT}

Introduction: Basketball is one of the sports that is most practiced at the collegiate, university, professional and amateur level combining acyclic actions and jumps during games, with high, medium and low intensities. For this reason, the power of the lower train in the vertical jumps used by athletes to win offensive, defensive rebounds and make throws to a basket of medium distance and 3 points, are a fundamental part of the development of this sport modality; consequently, the plyometric training method can be decisive for the increase of the power levels in the jumping capacity during the competition. Objective: To investigate the methods of plyometric training on the power of the lower train of the investigations already completed in basketball and analyze the result obtained according to it. Methodology: Is descriptive, since the interest of the author is to obtain accurate information to analyze and compare the effectiveness of plyometric training methods. Results: Articles, theses and reference books were based on plyometric training programs at national and international level in the modality of men's basketball that sustain positive effects on the power of the lower train according to the weeks planned by the research found. Conclusion: The bibliographic review in men's basketball aims to be a starting point to have advanced knowledge regarding plyometrics, lower train power, vertical jump, the instruments used and their effectiveness during training sessions in men's basketball.

Key words: Lower train power, Plyometrics, Basketball.

\section{INTRODUCCIÓN}

El baloncesto es uno de los deportes, que requiere caracterización de acciones explosivas y de potencia en el tren inferior en 
los saltos verticales (SV), además de una gran capacidad para ejecutar movimientos acíclicos por parte del jugador con una velocidad rápida de ejecución. En este sentido, es considerado un deporte intermitente acíclico, en el que se alternan períodos de actividad y de descanso, debido a que cuenta, con una gran variedad de acciones que ocurren de forma dinámica y continua como lo señala: Costa (2005); así mismo, este deporte ha venido en crecimiento en todo el mundo, porque es practicado en distintas edades y países, teniendo como base un deporte de alta exigencia que genera gran espectáculo y atracción del público. Además, es considerado una modalidad olímpica.

De igual modo, en el baloncesto los SV son fundamentales durante los encuentros deportivos, tanto en acciones defensivas y ofensivas; además, está relacionado con la potencia del tren inferior. Según un estudio realizado por: Romero, Vila, Ferragut, \& Alcaraz (2009), en el baloncesto, la habilidad de generar altos niveles de fuerza en cortos períodos de tiempo (potencia muscular), es un factor determinante para mejorar el rendimiento deportivo. Una forma de contribuir al rendimiento deportivo de los jugadores en el tren inferior, es realizar programas de entrenamiento pliométrico con una carga y velocidad de ejecución determinada para tener resultados efectivos en la potencia muscular inferior en cuanto al Ciclo de Estiramiento y Acortamiento (CEA).

En consecuencia, cuando una acción excéntrica precede a una concéntrica, la fuerza resultante de la acción concéntrica aumenta, ésa es la esencia del CEA (Brown \& Ferrigno, 2007). Para que el CEA sea efectivo se necesita un periodo de tiempo muy corto entre la fase excéntrica y la concéntrica, llamado tiempo de acoplamiento; cuanto menor sea éste, mayor energía adicional se conseguirá al almacenar en los elementos elásticos en serie (los tendones de los músculos) y en paralelo (formaciones de tejido conjuntivo que componen la membrana de las fibras musculares y sus haces) (Becerra \& Cáceres, 2004); algunos autores en sus argumentos mencionan que los métodos de entrenamiento pliométrico hacen parte del rendimiento deportivo en los jugadores de baloncesto, y fundamentando, la importancia de realizar trabajos de SV durante las sesiones de entrenamiento. Además, es uno de los métodos más adecuados y pertinentes utilizados por los entrenadores, para optimizar la potencia ofensiva y defensiva. Por otro lado, cabe discutir que, para medir la potencia del tren inferior en los jugadores, se utiliza una plataforma de contacto para realizar el Test de Bosco; de igual modo, también, existen test pedagógicos que ayudan a recopilar información en la potencia de los deportistas.

Además, durante los diversos encuentros competitivos se observa que los jugadores tienen una exigencia elevada en cambios de dirección y acciones de salto vertical SV. Por lo cual, al planificar un programa de entrenamiento pliométrico para mejorar la saltabilidad y la potencia, se crea una forma de mejorar las capacidades condicionales de los deportistas durante sus fases competitivas; así mismo, el baloncesto es un 
deporte donde se observan altos índices de intensidad de ida y vuelta durante los 40 minutos o más que dure el encuentro deportivo. Desde otro punto de vista, hay otro autor que menciona que la fuerza, ocupa un lugar esencial para cualquier ser humano, ya sea como capacidad física fundamental, limitante del rendimiento, o bien para garantizar la realización de cualquier acción motora (García, 1999, Siff \& Verhoshansky, 2000) y a su vez, en los deportes colectivos e individuales se habla que la potencia se refiere al producto entre la fuerza aplicada y la velocidad de la acción realizada" (Herzog \& Ait-Haddou, 2002). Por lo cual, el gesto técnico del SV en el baloncesto, también es importante practicarlo, porque influye en la capacidad de generar fuerza y conseguir una óptima velocidad de despegue. Durante la dinámica de juego de este gesto deportivo, que es el más preciso para fortalecer la capacidad de SV.

Por último, este artículo pretende encontrar resultados y diferencias efectivas en los métodos y programas de entrenamiento pliométrico en los jugadores de baloncesto, como hallazgo de una minuciosa revisión bibliográfica, Brown (1991): Se refiere a que: "el aparecimiento de nuevas estadísticas. Además, de expresar una evolución natural en el entendimiento de este proceso, está también, asociado a la necesidad que se ajustasen a las diferentes "filosofías de juego" de los entrenadores". Este apartado ayuda a los entrenadores a tener datos estadísticos más precisos y significativos, como base para realizar entrenamientos orientados a evolucionar el rendimiento deportivo durante las competencias.

\section{METODOLOGÍA}

El presente artículo es el resultado de una investigación con enfoque cualitativo con un diseño de investigación descriptivo, con el propósito de localizar los documentos bibliográficos descriptivos con criterios sobre, los programas de entrenamiento pliométrico y la potencia del tren inferior en jugadores de baloncesto masculino. En ese sentido, se encontraron varios documentos en la Biblioteca de la UPTC, entre libros, artículos de bases de datos y tesis de grado correlacionadas con respecto al baloncesto, por lo tanto, fueron analizados y validados a conveniencia del autor, donde se reflejaba información detallada sobre la temática a indagar.

\section{REVISIÓN TEÓRICA}

Durante el proceso de revisión bibliográfica, se encontraron documentos sobre los métodos de entrenamiento pliométrico en el tren inferior en baloncesto, en este apartado se observan algunas definiciones, una breve reseña histórica y tablas comparativas de investigaciones concluidas frente a la temática analizada. 
RESEÑA HISTÓRICA DE LA

PLIOMETRÍA.

El profesor Rodolfo Margaría durante la década de los 60, fue el primero en hablar de la relevancia del denominado ciclo estiramiento-acortamiento (CEA). El autor demostró que una contracción concéntrica precedida de una excéntrica, podía generar mayores niveles de fuerza que una contracción concéntrica aislada (Faccioni, 2001). Los trabajos del profesor Margaría fueron utilizados por la N.A.S.A. para desarrollar la manera más eficaz de caminar en la luna (Zanon, 1989). Este gran descubrimiento en el CEA, hizo que numerosos autores empezaran a investigar a más profundidad y creando nuevos conceptos, en cuanto a la pliometría. Al respecto Vladimir Zatsiorsky, fue quien utilizó en 1966 el término "pliométrico", buscando una tensión muscular excéntrica y concéntrica en el tren inferior de los deportistas. En ese mismo sentido, Yury Verkhoshansky, es considerado el padre de la pliometría aplicada al deporte, debido a que sus observaciones previas a los atletas de salto, lo llevaron a interesarse en desarrollar investigaciones en cuanto a la pliometría; de acuerdo a lo anterior, se dió a conocer un concepto mejor estructurado, por lo que, es preciso hacer un breve recuento de cómo nació, se desarrolló, evolucionó y qué importancia tiene este término en el siglo XXI, en especial para los entrenadores de las diferentes modalidades deportivas que dedican su tiempo a mejorar el rendimiento de las aptitudes físicas de los deportistas, con el propósito de revolucionar el deporte a nivel mundial.

Por otro lado, dentro de la conceptualización de los métodos de entrenamiento pliométrico, se deben tener en cuenta, conceptos que también han tenido gran trayectoria y avances en su línea de tiempo, por lo cual, son fundamentales como complemento del ciclo de acortamiento y estiramiento en los entrenamientos, siendo utilizados por los entrenadores deportivos y que se exponen a continuación:

\section{Fuerza}

Es uno de los factores importantes dentro del ámbito del baloncesto y cada día tiene mayor impacto dentro del entrenamiento deportivo, teniendo como propósito mejorar las habilidades y el desempeño de esta disciplina competitiva, además se habla de transferencia en situaciones y acciones de juego real, en consecuencia, González Badillo (2000), menciona que: "desde un punto de vista deportivo, se puede definir la fuerza como la manifestación externa (fuerza aplicada) que se hace de la tensión interna generada en el músculo o grupo de músculos en un determinado tiempo". La fuerza es una capacidad que debe ser incluida y trabajada dentro de las capacidades físicas para el buen rendimiento de los deportistas. 


\section{Velocidad}

Es una de las aptitudes físicas primordiales en el baloncesto, porque depende de una serie de movimientos coordinados incluyendo la elasticidad y la fuerza. Por otro lado, Ortiz (2004) define la velocidad como: "la capacidad de realizar movimiento ante un estímulo concreto, en el menor tiempo posible, con la mayor eficacia y donde la fatiga muscular durante los gestos técnicos y la ejecución no tiene un alto grado de concentración"; esta capacidad condicional aporta un rendimiento positivo a nivel individual y grupal, sí, es ejecutada de forma correcta. La velocidad, la rapidez y la capacidad de salto, son consideradas las facultades atléticas más valiosas en el baloncesto (Ben Abdelkrim, El Fazaa, \& El Ati, 2007), en ese mismo sentido, las capacidades fundamentales en las situaciones de juego real con o sin balón y su efectividad en la ejecución de dichos movimientos coordinados, dependerán de la potencia de despegue, la velocidad y la agilidad del jugador durante los ciclos competitivos.

\section{Potencia}

“La potencia se puede definir como la máxima cantidad de trabajo o de tensión muscular, que se puede desarrollar por una unidad de tiempo, o el producto de la fuerza por la velocidad" (Cronin \& Sleivert, 2005). Como expresa el autor, los grupos musculares durante el proceso deportivo son fundamentales. Además, el desarrollo adecuado de la potencia en el tren inferior en jugadores de baloncesto optimiza la calidad de los saltos del deportista y sube los niveles de efectividad durante los ciclos competitivos. Al respecto, Anselmi (2006) afirma que: "la potencia es la capacidad de realizar un trabajo en el menor tiempo posible y depende en forma directa de la fuerza y la velocidad" Al respecto las capacidades condicionales mencionadas anteriormente, cumplen aspectos importantes para subir los niveles de la pliometría en los deportistas.

\section{Pliometría}

“El término Pliométrico proviene del griego Plyethein, que significa "aumentar", y Metrique, que significa "Iongitud" (Wilt, 1978). En consecuencia, Cappa (2000) afirma que: la pliometría es un método de entrenamiento de la fuerza explosiva, utilizando componentes elásticos del músculo, para que el ciclo excéntrico de un movimiento sea más técnico al momento de ejecutarlo, y al mismo tiempo, se potencialice el ciclo concéntrico. Es importante, destacar que en los deportes colectivos como el baloncesto y el voleibol, es fundamental poder contar con la potencia del tren inferior, en los que, influye la capacidad de generar fuerza en un SV. Así mismo, Bompa (2005) la define como: "el músculo es cargado con una contracción excéntrica (estiramiento), seguido inmediatamente por una contracción concéntrica (acortamiento), mencionando 
que en la correcta realización de cada una de las fases el músculo se contraerá y extenderá más fuerte y rápido".

La pliometría es un método de entrenamiento utilizado para que los deportistas logren ejecutar la mayor fuerza y velocidad en el menor tiempo posible. Además se optimiza el tren inferior que brinda al jugador estabilidad y equilibrio al momento de realizar un salto vertical, también, el entrenamiento pliométrico obtiene otros beneficios como correr más rapido, reduce los niveles de fatiga, elimina calorías, fortalece los grupos musculares inferiores, incrementa la resistencia, entre otros factores, para mejorar la calidad física y técnica de los deportistas durante sus ciclos competitivos.

Sin embargo, la pliometría es un método de entrenamiento de la fuerza reactiva utilizada para optimizar los niveles de efectividad en el rendimiento de los jugadores, incrementando la velocidad y la saltabilidad de los deportistas. Dentro de la planificación pliométrica la fuerza es una capacidad muscular que debe ser orientada al rendimiento deportivo y a su vez, la asimilación neuro-funcional fisiológica (Barnes, 2003; Becerra \& Cáceres, 2004; Cometi, 2007), el entrenamiento proporciona que los músculos sean más explosivos durante su ejecución, al respecto, Verhoshanky (1999) define la capacidad reactiva como: "La capacidad específica de desarrollar un impulso elevado de fuerza inmediatamente después de un brusco estiramiento mecánico muscular; es decir, es la capacidad de pasar rápidamente del trabajo muscular excéntrico al concéntrico", así mismo, la pliometría se puede manifestar y educar con trabajos de baja intensidad con el objeto de fortalecer, estabilizar y potenciar la estructura anatómica del pie y el tobillo" (Zotko, 2002). Durante el proceso investigativo se pueden extraer varias conceptualizaciones, en cuanto a la importancia de realizar trabajos de entrenamiento pliométrico, porque los jugadores que practican deportes individuales o colectivos de forma competitiva optimizan el rendimiento deportivo y la capacidad de salto. Es evidente, que al combinar y ejecutar cada una de las fases mencionadas de forma correcta, se obtienen resultados positivos, avances tecnológicos y estadísticos en cuanto a las ciencias aplicadas del deporte.

En otro sentido, el baloncesto es un deporte que nació de la necesidad del hombre para practicar diversas actividades y juegos que estaban encaminados a lanzar pelotas, de acuerdo a estas acciones nacieron varios deportes y el baloncesto no es una excepción, sin embargo, a continuación, se expone una breve historia del baloncesto.

\section{Reseña Histórica de baloncesto.}

El baloncesto nació en 1891 en Estados Unidos, en la Universidad de YMCA, Springfield (Massachussets), fue allí donde el profesor Naismith, Licenciado en Educación Física, Recreación y Deportes, empezó a observar las prácticas deportivas que 
realizaban las personas durante el frío invierno (Rugby, atletismo, ciclismo, entre otros). Analizando los deportes del momento, surgió la idea de crear una modalidad deportiva innovadora que se pudiera realizar dentro de un recinto cubierto sin tener afecciones del frío. Todo comenzó con unas cestas de melocotones que el profesor colocó en los extremos de una pista a 3,05 metros del suelo, siendo ésta una altura adecuada para lanzar un balón y tratar de encestar. De allí, se originó el nombre de baloncesto, teniendo como objetivo fundamental: Introducir la pelota en las cestas de melocotones. Así mismo, el profesor también, ideó una normativa de juego que conoce esta modalidad deportiva como baloncesto (Webscolar, 2010).

Esta modalidad deportiva es reconocida en todo el mundo por millones de personas en la actualidad. Además, brinda un buen espectáculo para los aficionados, debido a que, los jugadores son conocidos por sus grandes habilidades individuales-grupales y aptitudes físicas, así mismo, su rendimiento y su efectividad en los lanzamientos a canasta durante los encuentros deportivos.

\section{Baloncesto}

Dentro del entorno, es cierto que es un deporte reglamentado y dinámico, que busca ser un juego más atractivo, adoptando con facilidad distintos niveles de aptitudes físicas y destrezas técnicas en las personas que lo practican; al respecto, Sampedro (1999) sustenta que, el baloncesto se sitúa dentro de los deportes definidos de cooperación / oposición. Donde la cohesión de grupo habita en los jugadores de equipo, aportando la consecución de un determinado objetivo. En consecuencia, la esencia del baloncesto es una pasión que irradia sensaciones de felicidad, integridad, expresión corporal, pedagogía, competitividad, entre otros, pero a su vez, se vuelve un gran océano iluminado de belleza y libertad, donde se disfruta el baloncesto en cada momento.

En consecuencia, se ilustra una tabla comparativa de las investigaciones de baloncesto culminadas, que son enfocadas a trabajos de entrenamiento pliométrico en jugadores; este es un registro fundamental para el desarrollo de esta revisión, y adicionalmente, se mostrará un programa pliométrico, que corresponde a un estudio realizado en E.E.U.U. En un apartado más adelante se observarán otras tablas comparativas de estudios realizados en otras modalidades deportivas. 
Tabla 1. Comparativa de la Revisión Bibliográfica en Baloncesto.

\begin{tabular}{|c|c|c|c|c|}
\hline Lugar, Año & Autor(es) & Título & $\begin{array}{l}\text { Entrenamientol } \\
\text { Método }\end{array}$ & $\begin{array}{c}\text { Resultados e } \\
\text { Incremento (salto } \\
\text { V) }\end{array}$ \\
\hline $\begin{array}{l}\text { España, } \\
\text { (2017): }\end{array}$ & $\begin{array}{l}\text { Sánchez, Floría, \& } \\
\text { Cardenal. }\end{array}$ & $\begin{array}{l}\text { Efecto del entrenamiento } \\
\text { combinado de fuerza y pliometría } \\
\text { en variables biomecánicas del salto } \\
\text { vertical en jugadores de } \\
\text { baloncesto. }\end{array}$ & $\begin{array}{l}\text { Entrenamiento } \\
\text { combinación de } \\
\text { fuerza y pliometría en } \\
\text { el salto vertical. }\end{array}$ & $12,02 \%$ \\
\hline $\begin{array}{l}\text { España, } \\
\text { (2016): }\end{array}$ & $\begin{array}{l}\text { Pedro Sánchez } \\
\text { González. }\end{array}$ & $\begin{array}{l}\text { Adaptaciones a un entrenamiento } \\
\text { integrado de fuerza, potencia y } \\
\text { propiocepción del tren inferior sobre } \\
\text { la estabilidad y el salto vertical en } \\
\text { baloncestor masculino } \\
\text { semiprofesional. }\end{array}$ & $\begin{array}{l}12 \text { Semanas de } \\
\text { Entrenamiento. }\end{array}$ & $P<0.05$ \\
\hline $\begin{array}{l}\text { México, } \\
\text { (2013): }\end{array}$ & $\begin{array}{l}\text { Édgar Eduardo } \\
\text { González García. }\end{array}$ & $\begin{array}{l}\text { El efecto de entrenamiento Anselmi } \\
\text { en el desarrollo de potencia y } \\
\text { velocidad en jugadores juveniles de } \\
\text { basquetbol. }\end{array}$ & $\begin{array}{l}12 \text { Semanas de } \\
\text { entrenamiento con } \\
\text { sobrecarga. }\end{array}$ & $P<018$ \\
\hline $\begin{array}{l}\text { Chile, } \\
\text { (2011): }\end{array}$ & $\begin{array}{lr}\text { Pedro } & \text { Delgado } \\
\text { Floody, Aldo Osorio } \\
\text { Poblete, } & \text { Rodrigo } \\
\text { Mancilla } & \text { Fuentes \& } \\
\text { Daniel } & \text { Jerez } \\
\text { Mayorga. } & \end{array}$ & $\begin{array}{l}\text { Análisis del desarrollo de la fuerza } \\
\text { reactiva y saltabilidad, en } \\
\text { basquetbolistas que realizan un } \\
\text { programa de entrenamiento } \\
\text { polimétrico. }\end{array}$ & 8 Semanas. & $P<0.05$ \\
\hline $\begin{array}{l}\text { Bogotá, } \\
\text { (2016): }\end{array}$ & $\begin{array}{l}\text { Leonardo } \\
\text { Rodríguez, Víctor } \\
\text { González \& Juan } \\
\text { Carlos. }\end{array}$ & $\begin{array}{l}\text { Programa pliométrico para } \\
\text { fortalecer la estabilidad articular en } \\
\text { miembros inferiores en los } \\
\text { jugadores de baloncesto Club The } \\
\text { Moles Categoría sub } 16 \text { de Bogotá. }\end{array}$ & $\begin{array}{l}8 \text { semanas } \\
\text { continuas. }\end{array}$ & $\begin{array}{l}\text { Pie derecho } 75 \% \\
\text { Pie izquierdo } 70 \%\end{array}$ \\
\hline
\end{tabular}

Fuente. Autoría propia.

La Tabla 1: Se realizó con el propósito de reflejar los documentos encontrados en baloncesto, durante el proceso de recolección de la información, que soporta el estado teórico de este artículo de revisión.

Por otra parte, en la Tabla 2: Muestra la estructura de un programa pliométrico, diseñado y planificado especialmente, para los jugadores de baloncesto, para subir los niveles de efectividad en la potencia del tren inferior. Los autores (Michael, Jeremy, Mark, Cristópher, \& Timothy, 2016, p. 10) que desarrollaron el estudio, sustentaron que el programa de entrenamiento produjo resultados significativos y positivos en la potencia de los deportistas. 
Tabla 2. Programa de Entrenamiento Pliométrico de 6 Semanas E.E.U.U.

\begin{tabular}{|c|c|c|c|c|}
\hline $\begin{array}{l}\text { Semana de } \\
\text { Entren. }\end{array}$ & $\begin{array}{l}\text { Vol. Entren. } \\
\text { (contacto de los } \\
\text { pies) }\end{array}$ & Ejercicio Pliométrico & $\begin{array}{c}\text { Series X } \\
\text { Rep. }\end{array}$ & $\begin{array}{l}\text { Int. De } \\
\text { Entren. }\end{array}$ \\
\hline \multirow{3}{*}{$\begin{array}{l}\text { Semana } \\
1:\end{array}$} & \multirow{3}{*}{90} & Saltos con los tobillos de un lado al otro. & $2 \times 15$ & Baja \\
\hline & & Saltar y alcanzar en el lugar. & $2 \times 15$ & Baja \\
\hline & & Saltos frontales a los conos. & $5 \times 6$ & Baja \\
\hline \multirow{4}{*}{$\begin{array}{l}\text { Semana } \\
\text { 2: }\end{array}$} & \multirow{4}{*}{120} & Saltos con los tobillos de un lado al otro. & $2 \times 15$ & Baja \\
\hline & & Salto en largo sin impulso. & $5 \times 6$ & Baja \\
\hline & & Saltos laterales sobre una barrera. & $2 \times 15$ & Media \\
\hline & & Saltos con dos piernas. & $5 \times 6$ & Media \\
\hline \multirow{5}{*}{$\begin{array}{l}\text { Semana } \\
\text { 3: }\end{array}$} & \multirow{5}{*}{120} & Saltos con los tobillos de un lado al otro. & $2 \times 12$ & Baja \\
\hline & & Salto en largo sin impulso. & $4 \times 6$ & Baja \\
\hline & & Saltos laterales sobre una barrera. & $2 \times 12$ & Media \\
\hline & & Saltos con dos piernas. & $3 \times 8$ & Media \\
\hline & & Saltos laterales a los conos. & $2 \times 12$ & Media \\
\hline \multirow{5}{*}{$\begin{array}{l}\text { Semana } \\
\text { 4: }\end{array}$} & \multirow{5}{*}{140} & Saltos diagonales a los conos. & $4 \times 8$ & Baja \\
\hline & & Salto en largo sin impulso con sprint lateral. & $4 \times 8$ & Media \\
\hline & & Saltos laterales a los conos. & $2 \times 12$ & Media \\
\hline & & Saltos con una sola pierna. & $4 \times 7$ & Alta \\
\hline & & Saltos laterales con una sola pierna. & $4 \times 6$ & Alta \\
\hline \multirow{6}{*}{$\begin{array}{l}\text { Semana } \\
\text { 5: }\end{array}$} & \multirow{6}{*}{140} & Saltos diagonales a los conos. & $2 \times 7$ & Baja \\
\hline & & Salto en largo sin impulso con sprint lateral. & $4 \times 7$ & Media \\
\hline & & Saltos laterales a los conos & $4 \times 7$ & Media \\
\hline & & Saltos a los conos con giros de 180 grados & $4 \times 7$ & Media \\
\hline & & Saltos con una sola pierna & $4 \times 7$ & Alta \\
\hline & & Saltos laterales con una sola pierna & $2 \times 7$ & Alta \\
\hline \multirow{5}{*}{$\begin{array}{l}\text { Semana } \\
6:\end{array}$} & \multirow{5}{*}{120} & Saltos diagonales a los conos & $2 \times 12$ & Baja \\
\hline & & Salto hexagonal & $2 \times 12$ & Baja \\
\hline & & $\begin{array}{l}\text { Saltos a los conos con sprint con cambio de } \\
\text { dirección }\end{array}$ & $4 \times 6$ & Media \\
\hline & & Saltos con dos piernas & $3 \times 8$ & Media \\
\hline & & Saltos laterales con una sola pierna & $4 \times 6$ & Alta \\
\hline
\end{tabular}

Fuente. Autoría propia.

A continuación, se sustenta uno de los conceptos importantes para el desarrollo del salto en los jugadores de baloncesto, debido a que la pliometría es mencionada como uno de los métodos más efectivos para ganar potencia y saltabilidad, teniendo en cuenta, que en el baloncesto los deportistas deben tener gran capacidad para saltar lo más alto posible, y en el momento preciso, siendo uno de los aspectos importantes dentro de las acciones específicas del juego como los rebotes, los tapones o los lanzamientos en suspensión (Tous, 2008). 
De acuerdo a los estudios reflejados en la Tabla 1 del artículo, los investigadores dentro de la literatura utilizaron como medio de recolección de datos: El Test de Bosco. La plataforma de contacto. Además, se observaron en los documentos varios medios para la recolección de los datos estadísticos, con el objeto de evaluar la potencia del tren inferior y poder desarrollar los programas de entrenamiento pliométricos para cada uno de los deportes.

\section{DISCUSIÓN}

En el proceso de revisión bibliográfica, se identificaron documentos con aplicación de los métodos del entrenamiento pliométrico para el tren inferior en deportista jugadores de baloncesto, durante esta revisión teórica, se observaron definiciones y reseñas históricas significativas, además, de tablas comparativas de investigaciones concluidas frente a la temática analizada. El baloncesto es uno de los deportes que más se practica a nivel colegiado, universitario, profesional y amateur, combinando acciones acíclicas y saltos durante los partidos, con intensidades altas, medias y bajas.

La potencia del tren inferior en los saltos verticales utilizados por los deportistas para ganar rebotes ofensivos, defensivos y realizar lanzamientos a la canasta de media distancia $y$ a tres (3) puntos, son una parte fundamental dentro del desarrollo de esta modalidad deportiva; por ende, el método de entrenamiento pliométrico puede ser determinante para el incremento de los niveles de la potencia en la capacidad de salto durante la competencia.

Se fundamenta que los resultados que se identificaron son provenientes de las bases de datos, tesis y libros correlacionados con programas de entrenamiento pliométrico a nivel nacional e internacional en la modalidad de baloncesto masculino, que sustentan efectos positivos en la potencia del tren inferior de acuerdo a las semanas planificadas por las investigaciones revisadas. El resultado de esta revisión teórica con enfoque cualitativo y con un diseño de investigación descriptivo, facilitó el propósito de rastrear e identificar artículos y variedad de documentos bibliográficos descriptivos con criterios sobre: programas de entrenamiento pliométrico y la potencia del tren inferior en jugadores de baloncesto masculino.

\section{CONCLUSIONES}

De acuerdo a la revisión bibliográfica en la potencia del tren inferior, en las investigaciones de baloncesto de la Tabla 1, se sustenta que dos investigaciones tuvieron datos de significancia similares de $p<0.05$ a pesar que uno tiene un programa de entrenamiento de 12 semanas y el otro de 8 semanas. Sin embargo, otros estudios muestran hallazgos de $p<0.18,12,02 \%$ y un último en $75 \%$ en el pie derecho y $70 \%$ en el pié izquierdo. En ese sentido, los programas de entrenamiento pliométrico, según las semanas establecidas reflejan niveles de 
optimización y rendimiento en la potencia del tren inferior en los deportistas.

En consecuencia, en los estudios analizados se menciona que los programas de entrenamiento pliométrico del tren inferior para desarrollar potencia en los saltos verticales, tienen un grado de significancia de acuerdo al número de saltos que se realizan durante las sesiones de entrenamiento a la semana en los deportistas. Con relación a los métodos de entrenamiento pliométrico, es posible optimizar las manifestaciones de la fuerza y la velocidad explosiva, para obtener mejoras en la potencia del tren inferior en los jugadores de baloncesto.

\section{REFERENCIAS BIBLIOGRÁFICAS}

ANSELMI, H. 2006. Actualización sobre Entrenamiento de la potencia. Argentina: RyC Editora. Décima Edición.

ARENAS, J. 2009. Influencia de un plan de seis semanas de entrenamiento pliométrico de moderada intensidad en miembros inferiores sobre el índice elástico de las jugadoras de voleibol femenino de la Institución Educativa INEM José Félix de Restrepo, edades de 14 y 17 años. Medellín/Antioquia: Universidad de Antioquía.

ARENAS, M., \& ARANGO, O. 2016. Programa de entrenamiento para el remate en fútbol sala en categoría juvenil de Selección Chía, Bogotá/Colombia: Universidad Pedagógica Nacional.

BARNES, M. 2003. Introducción a la Pliometría. [Electronic Versión]. Sobre entrenamiento from. Obtenido de http://www.sobreentrenamiento.com/PubliCE/ Articulo.asp?ida=213\&tp=s.

BECERRA, H., \& CÁCERES, Z. 2004. La pliometría, más que una técnica de multisaltos. Efdeportes Revista Digital -.

BECERRA, R., \& CÁCERES, B. 2004. Pliometría, más que una técnica de Multisaltos. [Electronic Versión]. Obtenido de http://www.efdeportes.com/, from http:// www.efdeportes.com/efd73/pliom.htm.

BEDOYA, J. 2009. Efectos de un plan de entrenamiento de pliometría mediante saltos en vallas y fuerza máxima de miembros inferiores en futbolistas juveniles. Medellín/Colombia: Universidad de Antioquia.

BEN ABDELKRIM, N., EL FAZAA, S., \& EL ATI, J. 2007. Time-motion analysis and physiological data of elite under-19-year-old basketball players during competition. British journal of sports medicine, 41(2), 69-75.

BOMPA, T. 2005. Periodización de la fuerza. La nueva onda de entrenamiento de la fuerza. Grupo sobre Entrenamiento. Primera Edición Digital. 
BROWN. 1991. Staying fit and staying well: Physical fitness as a moderator of life stress. Journal of Personality and Social Psychology, 60, 555-561.

BROWN, L., \& FERRIGNO, V. 2007. Entrenamiento de la velocidad, agilidad y rapidez. Barcelona: Editorial Paidotribo.

BROWN, M., MAYHEW, J., \& BOLEACH, L. 1986. Effect of plyometric training on vertical jump performance in high school basketball players. J. Sports Med. Phys. Fitness. 26(1):1-4.

CAPPA, D. (2000). Entrenamiento de la Potencia Muscular. Grupo sobre Entrenamiento. Primera Edición Digital.

COMETI, G. 2007. Los métodos Pliométricos. (PubliCE Standard,). [Electronic Versión]. Obtenido de: http:// www.sobreentrenamiento.com/publice/Articulo. asp?ida=848

COSTA, I. 2005. Características Físico-Fisiológicas de los jugadores de Basquetbol. Obtenido de ([Electronic Versión]). Obtenido de PubliCE Standard, from http://www.sobreentrenamiento.com/PubliCE/Articulo.asp?ida=466\&tp=s.

CRONIN, J., \& SLEIVERT, G. 2005. Challengers in understanding the influence of maximal power training on imporving athletic performance. Sport Medicine, 35(3),213-234.

DELGADO, P., OSORIO, A., MANCILLA, R., \& JEREZ, D. 2011. Análisis de desarrollo de la fuerza reactiva y saltabilidad, en basquetbolistas que realizan un programa de entrenamiento pliométrico. Motricidad y persona, 12.

DIALLO, O., DORE, E., DUCHE, P., \& VAN PRAAGH, E. 2001. Effects of plyometric training followed by a reduced training programme on physical performance in prepubescent soccer players. J. Sports Med. Phys. Fitness., 41(3): 342-348.

FACCIONI, A. 2001. Plyometrics. <http://www.faccioni.com/reviews/pliometrics.htm > [Consulta: 21/09/02].

FATOUROS, I., JAMURTAS, A., LEONTSINI, D., TAXILDARIS, K., AGGELOUSIS, N., KOSTOPOULOS, N., \& BUCKENMEYER, P. 2000. Evaluation of plyometric exercise training, weight training, and their combination on vertical jumping performance and leg strength. Journal Strength and Conditioning Research, 14 (4): 470-476.

FIBA (2018). Reglas Oficiales del Baloncesto. Barcelona, España: FIBA.

FLARITY, J., SHILSTONE, M., IGLESIA, T., \& FISHER, Z. 1997. The effectiveness of the Strengt Shoe ${ }^{\circledR}$ in enhancing sport specific skills. Obtenido de <http:// www.strengthsystems.com/strshoes.php>. [Consulta: 04/12/01].

GARCÍA, F., \& PEÑA, J. 2016. Efectos de 8 semanas de Entrenamiento Pliométrico y Entrenamiento resistido mediante Trineo en el rendimiento de Salto Vertical y Esprint en Futbolistas Amateurs. Sport Performance Analysis Research Group.

GARCÍA, M. 1999. "La Fuerza Fundamentación, valoración y entrenamiento". Madrid, España: Editorial Gymnos.

GONZÁLEZ BADILLO, J. 2000. Concepto y media de la fuerza explosiva en el deporte. Posibles aplicaciones al entrenamiento. Revista de Entrenamiento Deportivo, 14(1), 5-16. 
GONZÁLEZ, E. 2013. El efecto de entrenamiento Anselmi en el desarrollo de la potencia y velocidad en jugadores juveniles de basquetbol. México: Universidad Autónoma de Nuevo León.

GORDILLO, S., BENÍTEZ, D., ACOSTA, P., \& SANABRIA, Y. 2018. Fuerza explosiva y agilidad en jugadores de baloncesto. Revista Digital: Actividad Física Y Deporte, 5(1), 5-14. https://doi.org/10.31910/rdafd.v5.n1.2019.1117

HAKKINEN, K., \& KOMI, P. 1985. Effect of explosive strength training on electromyographic and force production characteristics of legs extensor. Scand. Jour. Sports Sci., 7(2): 65-76.

HERZOG, W., \& AIT-HADDOU, R. 2002. Mechanical muscle models and theri application to force and power production, chaper 9. In K. P.V.: Strength and power in sport. $2^{\circ}$ ed., pp. 154-183: Blackwell Science.

MARTÍNEZ, W. 2019. Una revisión bibliográfica sobre la pliometría en el tren inferior en baloncesto. Tunja, Boyacá.

MARTÍNEZ, A., MIRA, J., CUESTA, B., PÉREZ, J., \& ALCARAZ, P. 2017. La pliometría en el Voleibol Femenino. Revisión Sistemática. Federación Española de Asociaciones de Docentes de Educación Física, 7.

MATAVULJ, D., KUKOLJ, M., UGARKOVIC, D., TIHANYI, J., \& JARIC, S. 2001. Effects of plyometric training on jumping performance in junior basketball players. J. Sports Med. Phys. Fitness, 41(2):159-164.

JEREMY, J., MARK, D., CRISTÓPHER, C., \& TIMOTHY, J. 2016. Efecto de un programa de entrenamiento pliométrico de seis semanas sobre la agilidad. Entrenamiento Deportivo, p. 10.

MUÑEZ, A. 2016. Pliometría contextualizada en el Fútbol y el Baloncesto. Mejoras esperadas vs reales. Sportis Scientific Technical Journal, p. 22.

OCHOA, G. 2013. Desarrollo de la potencia muscular en el rendimiento deportivo para la selección de baloncesto en la categoría varones (15-16 años) de la Federación Deportiva estudiantil de Santa Elena (FEDEPESE), Provincia de Santa Elena. La Libertad/Ecuador: Universidad Estatal Península de Santa Elena.

OROZCO \& TODOROV. 2008. Técnica en: Orozco y Todorov. Guía del baloncesto. COLDEPORTES. Bogotá: COLDEPORTES, pp. 1-39.

ORTIZ, R. 2004. Tenis: Potencia, Velocidad y Movilidad. Editorial INDE.

PÉREZ, J. 2015. Propuesta metodológica para la aplicación de los niveles 0, 1 y 2 de Pliometría en Futbolistas Amateur. Punta de Arenas/Chile: Universidad de Magallanes.

QUETGLAS, Z., IGLESIA, O., \& MARTíNEZ, R. 2012. Fundamentos Biomecánicos del ejercicio pliométrico. Educación Física y Deportes, Revista Digital.

RODRÍGUEZ, L., GONZÁLEZ, V., \& RAMÍREZ, J. 2016. Programa pliométrico para fortalecer la estabilidad articular de miembros inferiores de los jugadores de baloncesto Club The Mole categoría sub 16 de Bogotá. Bogotá: Corporación Universitaria Minuto de Dios. 
ROMERO, S., VILA, H., FERRAGUT, C., \& ALCARAZ, P. 2009. Curva de potencia en jugadores de baloncesto de la Liga EBA. Obtenido de Recuperado el 31 de agosto del 2019, de http://redalyc.uaemex.mx/src/inicio/ArtPdfRed.jsp?iCve=227014933019\#.

SAMPEDRO, J. 1999. Fundamentos de la Táctica deportiva. Análisis de la estrategia de los deportes. Madrid, España: Editorial Gymnos.

SÁNCHEZ, P. 2016. Adaptaciones a un entrenamiento integrado de fuerza, potencia y propiocepción del tren inferior sobre la estabilidad y el salto vertical en baloncesto masculino semiprofesional. España/Murcia: Universidad Autónomo de Nuevo León.

SÁNCHEZ, A., FLORIA, P., \& CARDENAL, S. 2017. Efecto del entrenamiento combinado de fuerza y pliometría en variables biomecánicas del salto vertical en jugadores de baloncesto. Federación Española de Asociaciones de Docentes de Educación Física, p. 4.

SIFF, M., \& VERHOSHANSKY, Y. 2000. Super Entrenamiento. Barcelona, España: Editorial Paidotribo.

SPURRS, R., MURPHY, A., \& WATSFORD, M. 2003. The effect of plyometric training on distance running performance. Eur. J. Appl. Physiol. 89(1):1-7.

TORRES, A., CARMONA, J., \& STEPHENS, R. 2005. Efectos del entrenamiento pliométrico en miembros superiores en la fuerza aplicada y la precisión de tiro de la bola, con jóvenes beisbolistas de 12 a 14 años de edad del Inder Envigado. Medellín/Colombia: Universidad de Antioquia.

TOUS, J. 2008. Entrenamiento de la Fuerza en Baloncesto. En Terrados, N. y Calleja, J. (Coor.), Fisiología, Entrenamiento y Medicina del Baloncesto. Barcelona, España: Editorial Paidotribo, pp. 163-174.

VERHOSHANKY, Y. 1999. Todo sobre el método pliométrico. Capítulos 1 y 2. Barcelona: Paidotribo.

WILSON, G., NEWTON, R., MURPHY, A., \& HUMPHRIES, B. 1993. The optimal training load for the developement of dynamic athletic performance. Med. Sci. Sports Exerc., 25(11): 12791286. .

WILT, F. 1978. Plyometrics: What it is and howw it works. Modern Athlete an Coach, 16: 9-2. ZANON, S. 1989. Plyometrics: past and present. New Studies In Athletics, 4: 7-17. ZOTKO, R. 2002. Specific Excercises for Tendons and Ligaments. Modern Athlete and Coach, 40(3), 37-40.

Cómo citar: Martínez, W., López, F., Acosta, P., \& Sanabria, Y. (2019). Una mirada bibliográfica sobre la influencia de la pliometría en el tren inferior en baloncesto. R. Actividad fis. y deporte. 6 (1): 179-193. 